\title{
Prioritization and management recommendations of pediatric urology conditions during the COVID-19 pandemic
}

\author{
Daniel T. Keefe, $M D^{1 *}$; Mandy Rickard, $M N$, $N P^{1 *}$; Peter Anderson, $M D^{2}$; Darius Bagli, $M D^{1}$; \\ Anne-Sophie Blais, MD'; Stéphane Bolduc, MD"; Luis H. Braga, MD, PhD5; Natasha Brownrigg, MN, NP5; \\ Michael Chua, MD'; Sumit Dave, MD'; Joana dos Santos, MD'; Luis Guerra, MD'; Allen H. Hayashi, MD, MSc; \\ Mélise A. Keays, MD'; Soojin Kim, MD'; Martin A. Koyle, MD, MSc'; Linda C. Lee, MD'; \\ Armando J. Lorenzo, MD, MSc'; Dawn MacLellan, MD'; Landan MacDonald, MD'; Andrew E. MacNeily, MD'; \\ Peter D. Metcalfe, MD, MSc ${ }^{10}$; Katherine Moore, $M D^{4}$; Rodrigo L.P. Romao, MD ${ }^{2,3}$; Peter Z.T. Wang, $M D^{6}$
}

${ }^{\star}$ Co-first authors

'Division of Urology, The Hospital for Sick Children, Toronto, ON, Canada; ${ }^{2}$ Division of Pediatric Urology, IWK Health Centre, Department of Urology, Dalhousie University, Halifax, NS, Canada; ${ }^{3}$ Division of Pediatric Surgery, IWK Health Centre, Department of Urology, Dalhousie University, Halifax, NS, Canada; ${ }^{\circ}$ Division d'Urologie, Département de Chirurgie, CHU de Québec, Quebec City, QC, Canada; ${ }^{5}$ Department of Surgery/Urology, McMaster University, Hamilton, ON, Canada; ${ }^{6}$ Department of Surgery, Division of Urology, Western University, London, ON, Canada; ${ }^{7}$ Department of Surgery, Division of Pediatric Urology, Children's Hospital of Eastern Ontario, University of Ottawa, Ottawa, ON, Canada; ${ }^{8}$ Division of Pediatric Surgery, Department of Surgery, Victoria General Hospital, Victoria, BC, Canada; ${ }^{9}$ Department of Urologic Sciences, University of British Columbia, Vancouver, BC, Canada; ${ }^{10}$ Department of Surgery, Division of Pediatric Surgery, University of Alberta, Stollery Children's Hospital, Edmonton, AB, Canada

Cite as: Can Urol Assoc J 2020;14(6):E237-50. http://dx.doi.org/10.5489/cuaj.6693

\section{Introduction}

The declaration of a worldwide pandemic related to the novel coronavirus (Sars-CoV-2) by the World Health Organization on March 11, 2020 ${ }^{1}$ has led to significant disruptions in the daily life of Canadians. In an attempt to minimize the spread of coronavirus disease 2019 (COVID-19), restrictions on public gatherings have been implemented. Widespread public adoption of social distancing regulations seems to have alleviated some of the burden on the healthcare system in what epidemiological experts have promoted as "flattening the curve." ${ }^{2}$

In an attempt to comply with legislated distancing measures, healthcare institutions have made dramatic changes to the care of patients during the pandemic, including the indefinite postponement of all non-essential clinic visits and elective surgeries. This significant and unprecedented change in practice has pushed healthcare providers to find creative and alternative strategies to ensure patients continue to receive the care they need while minimizing risk to both providers and patients and respect the distancing measures. The Canadian Urological Association Journal (CUAJ) has published a decision-making algorithm on how to prioritize elective urology surgeries in an attempt to standardize management, protect hospital resources, and, ultimately, mitigate the impact of COVID-19 on the outcomes of those we treat. ${ }^{3}$
Multiple studies across several medical specialties have shown that telehealth is feasible and safe, and can have positive impacts on healthcare resources, patient quality of life, and the environment. ${ }^{4-7}$ Urologists have also explored the use of telemedicine for patients with ureteral colic and have shown promising outcomes, including an improvement in clinic capacity and financial benefits without sacrificing care. ${ }^{8,9}$ Pediatric urology patients range from those with non-urgent concerns that affect the urogenital tract to those with life-threatening conditions. Children with serious urological concerns will need to be managed urgently despite the current pandemic, both in the clinic and the operating room. However, many of the less serious conditions may be amenable to virtual visits or telephone consultations.

\section{Methods}

In order to determine how Canadian pediatric urology patients are being managed during the COVID-19 pandemic, as well as to provide some guidance to providers, pediatric urology clinicians from across Canada were invited to provide expert recommendations on the clinical management of pertinent conditions. Participants were asked to complete a set of standardized questions related to a specific pediatric urological diagnosis (Table 1). Experts were also asked to stratify patients into four possible rebooking priorities based on urgency (Table 2). Table 3 summarizes the conditionrelated rebooking priority once the pandemic is resolved. The objective of the commentary is to provide a document 


\begin{tabular}{l}
$\begin{array}{l}\text { Table 1. Standard questions provided to manuscript } \\
\text { authors }\end{array}$ \\
$\begin{array}{cc}\text { How has your triaging of this condition been adjusted given } \\
\text { the COVID-19 pandemic? }\end{array}$ \\
How do you anticipate triaging of this condition will be \\
adjusted once pandemic restrictions are lifted? \\
Are there any initial investigations and management \\
$3 \quad \begin{array}{c}\text { Are there any red flag signs or symptoms that would } \\
\text { prompt more immediate assessment and }\end{array}$ \\
management of this patient? \\
$4 \quad \begin{array}{c}\text { Can this patient be adequately assessed via telehealth? If not, } \\
\text { what rebooking priority would you assign this patient? } \\
\text { Do you have any recommendations regarding how to } \\
\text { mitigate concerns to referring providers or parents/patients } \\
\text { of delayed assessment and management of this condition? }\end{array}$ \\
\hline
\end{tabular}

that can be referenced by primary care providers, as well as general and pediatric urologists, in order to help standardize the care of pediatric urology patients during the pandemic and provide guidance on managing the surge of patients once restrictions begin to be lifted.

\section{Hydronephrosis}

(Authors: Mandy Rickard, NP; Dr. Michael Chua)

\section{Background}

Hydronephrosis $(\mathrm{HN})$ is one of the most common congenital anomalies detected prenatally, affecting $1-5 \%$ of pregnancies. ${ }^{10}$ Determining the etiology of $\mathrm{HN}$ requires further diagnostic investigations after birth. ${ }^{10,11}$ Hence, pediatric urology providers may be asked to participate in prenatal counselling and management of patients with congenital anomalies of the kidneys and urinary tract (CAKUT). During the COVID-19 pandemic, while it may be possible to postpone the assessment of many urological conditions, pre- and postnatal CAKUT may require counselling, assessments, and investigations.

\section{Conditions to screen for and red flag signs/symptoms}

- Male infants with concern for posterior urethral valves (PUV)

- Bilateral hydroureteronephrosis $(\mathrm{HUN}) \pm$ bladder abnormalities

- Solitary kidney with severe HN

- Dysplastic kidneys

\section{Prenatal consultations}

Antenatal anterior-posterior diameter (APD) $>15 \mathrm{~mm}$ in the third trimester is associated with postnatal pathology, ${ }^{12}$ and CUA guidelines on the management of antenatal $\mathrm{HN}$ rec-

\begin{tabular}{lc}
$\begin{array}{l}\text { Table 2. Recommendations for rebooking priority for } \\
\text { patients amenable to delayed clinical visits after virtual } \\
\text { visit confirms no significant change in status }\end{array}$ \\
\begin{tabular}{lc} 
Rebooking priority & Recommendation \\
\hline $\begin{array}{l}\text { Priority } 1 \\
\text { Priority } 2\end{array}$ & Urgent: Rebook as soon as possible \\
Priority 3 & Nomi-urgent: Safe to postpone clinic visit up \\
to 3 months
\end{tabular} \\
$\begin{array}{c}\text { Priority } 4 \\
\text { Elective: Safe to postpone clinic visit beyond } \\
\text { to } 6 \text { months }\end{array}$ \\
\hline
\end{tabular}

ommend referral to a specialized center for APD $>7 \mathrm{~mm} \cdot{ }^{13}$ Prenatal consultations are amenable to virtual visits, as review of imaging can be carried out remotely and consultations can be completed via video/teleconferencing. Diagnostic findings can be reviewed with the expectant parents, as well as discussions on the postnatal recommendations, including additional investigations (followup ultrasound [US], voiding cystourethrogram [VCUG], and indications for continuous antibiotic prophylaxis [CAP]). ${ }^{13,14}$

\section{Postnatal CAKUT triage recommendations}

\section{Urgent, in-person clinic assessment}

- Severe HN (Society for Fetal Urology [SFU] grade 3-4, APD $>15 \mathrm{~mm}$ )

- Bilateral HN

- HN in a solitary kidney

- Suspected PUV

- Duplex system with HUN, including suspected ureteroceles

- Postoperative visits requiring procedures (i.e., stent removals)

- Postoperative complications (infections; pain)

- Complex patients with a change in status (e.g., PUV patient with decreased urine output)

\section{Postponed in-person clinic assessment}

- Infants with mild HN (SFU grade 1-2, APD 7-10 mm)

- Moderate unilateral HN (SFU grade 3)

- Vesicoureteral reflux (VUR) on CAP

- Duplication anomalies, voiding well, and infection-free with CAP

- Followup visits with stable dilatation and no urinary tract infections (UTIs)

- Postoperative patients who are progressing well at home For patients with US findings that are suggestive of a condition associated with a higher risk of infections (i.e., high-grade $\mathrm{HN}$, hydroureter), CAP should be considered until appropriate imaging and in-person assessment can take place. 


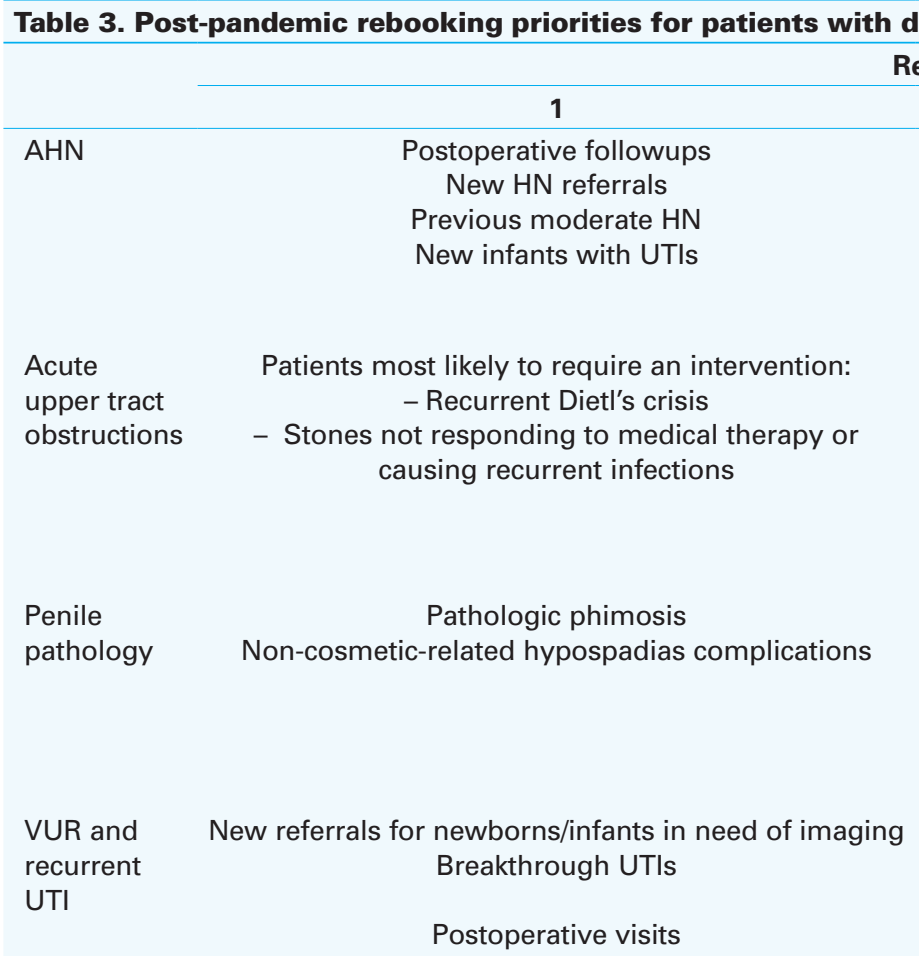

Inguinal pathology
BBD

Neurogenic bladder

Testicular and fertility concerns

\section{Abnormal upper tracts on ultrasound Breakthrough febrile UTIs Abnormal renal function Persistently high PVR}

DSD

New suspicion of DSD later in childhood whose visit was virtual and a physical examination was not completed

New DSD diagnosis that for some reason was not seen by the pediatric urology team at birth in the hospital Postoperative followups

HN/HUN; febrile UTIs; asymptomatic kidney and bladder stones; solitary kidneys; HN/HUN Difficulty catheterizing channel/stomal stenosis Infants ( $<12$ months) for surveillance of bladder function/upper tracts

Gradual changes to voiding patterns

Postoperative visits for recent reconstructions Bilateral UDT Non-palpable testes Symptomatic varicoceles $>24$ months with UDT
History of Dietl's crisis but are not experiencing repeated episodes

Postoperative patients who have not had a followup visit

Stable stones

Routine postoperative followups

\section{Routine followups with imaging}

Infants/non-toilet-trained children on CAP with no UTIs

New patients with suspected inguinal hernias Children >24 months with hydroceles

$\geq 6$ months unsuccessful bladder retraining

Associated VUR with CAP

Refractory patients on

medication or alternative therapies

Patients with a delayed surgical intervention

Children 12-24 months with bladder emptying regimens VUR and taking CAP

Older children with bladder emptying regimens; previously normal upper tracts

12-24 months with UDT Asymptomatic varicoceles Postoperative followups
New hypospadias consultations

Hypospadias who have undergone the first stage of a staged repair

Post CAP DC with

no UTI + previously normal/stable ultrasound

Older children on CAP with no UTIs

New referrals for boys $<24$ months with hydroceles

Already have had at least one consultation and are progressing well

Stable DSD patients whose visits were deferred
New referrals for boys $<12$ months with hydroceles

Stable patients with annual visits and are close to discharge to primary care
Older children with bladder emptying regimens; previously normal upper tracts

\section{6-12 months with UDT}

Routine surveillance with stable imaging 
Keefe et al

Table 3 (cont'd). Post-pandemic rebooking priorities for patients with deferred clinic visits

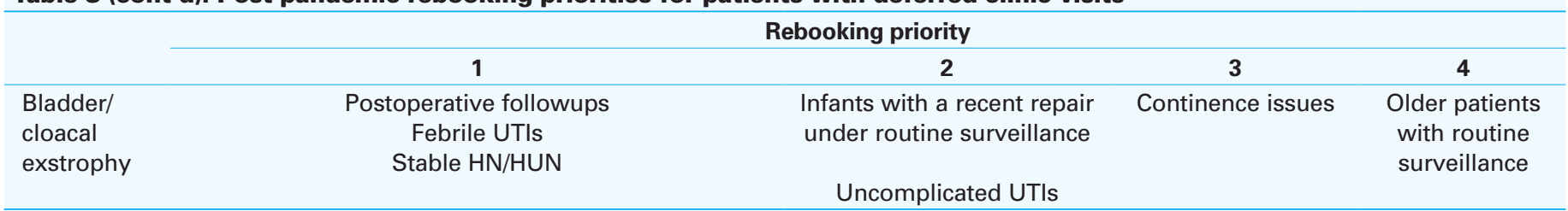

AHN: antenatal hydronephrosis; BBD: bladder and bowel dysfunction; CAP: continuous antibiotic prophylaxis; DSD: disorder of sexual differentiation; HN: hydronephrosis; HUN: hydroureteronephrosis; PUV: posterior urethral valves; US: ultrasound; UTI: urinary tract infection; VUR: vesicoureteral reflux.

\section{Virtual visit/telephone encounter (pre- and postnatal CAKUT)}

A careful history taken from the family and the prenatal care team is critical. Information regarding the prior antenatal diagnostic findings, family medical history, and background are important pieces of information during counselling. Discussion of the diagnostic findings and management plan depends on the spectrum of CAKUT, prognosis, and families' cultural background. However, it is important to clarify the risk-benefit of each option, such as continuous monitoring (with/without diagnostics), consideration of circumcision for parents of newborn males (as this can be completed prior to discharge), as well as CAP when indicated.

\section{Investigations to be obtained prior to assessment}

Prenatal parental counselling: Antenatal US(s) for review, including information on essential measurements, such as APD, amniotic fluid index, laterality, presence of bladder abnormalities and suspected anomalies to determine the severity and etiology of $\mathrm{HN}$.

Postnatal: US for assessment of HN severity. This is particularly important for newborn males with bilateral $\mathrm{HN}$ to document indicators of PUV (bilateral hydroureter, bladder abnormalities, dilated posterior urethra). The necessity of VCUG should be determined by a pediatric urology provider upon review of initial ultrasounds. Infants with signs of dysplastic kidneys, solitary kidney with $\mathrm{HN}$, or with increased renal echogenicity should also be investigated with baseline laboratory investigations (electrolytes and kidney function tests) prior to the initial consultation followed by a referral to pediatric nephrology.

\section{Management}

Postnatally, communication between the obstetrics provider and the pediatric urology team is important, particularly for newborns with findings that meet the criteria for urgent visits (see above). Requests for followup imaging, placement of a catheter, and/or CAP may need to be discussed prior to discharge from the hospital.

\section{Acute upper urinary tract obstruction (Author: Dr. Peter Metcalfe)}

\section{Background}

Patients with $\mathrm{HN}$ may be categorized into symptomatic and asymptomatic, with the understanding that the presence of symptoms refers to the probability of acute obstruction. The most common etiologies of acute upper tract obstructions in children and adolescents are calculi and ureteropelvic junction obstructions (UPJO). Patients may present to the emergency department (ED) with severe flank pain and/or vomiting due acute UPJO in a syndrome known as Dietl's crisis. Due to limited access to radiology during the COVID19 pandemic, most routine followup imaging is not possible and alternative assessments must be conducted. However, postponement of every case may not be feasible. Ultimately, with respect to management of symptomatic urinary tract obstruction, as a semi-urgent to urgent illness, acute treatment should not differ due to pandemic restrictions.

\section{Conditions to screen for and red flag signs/symptoms}

- Obstructive stones with symptoms (infection, pain, elevated kidney function markers)

- Symptomatic UPJO (flank pain, vomiting, without spontaneous improvement, infection)

- Evidence of obstruction in a solitary kidney

\section{Triage recommendations}

\section{Urgent, in-person clinic assessment}

- Pyelonephritis/sepsis in an obstructed system

- Pain, nausea, and/or vomiting not resolving with optimal medical management

- Traumatic rupture of a hydronephrotic kidney

- Obstructive calculi that may benefit from intervention for relief of symptoms and to prevent further visits to the ED

- Postoperative patients requiring a procedure such as stent removal 


\section{Postponed in-person clinic assessment}

- Well children with no UTI or further episodes of Dietl's crisis

- Response to medical management (pain management for intermittent flank pain, expulsion therapy for calculi)

- Postoperative children who are progressing well without change in symptoms

- Known non-obstructing stones that remain asymptomatic

\section{Virtual visit/telephone encounter}

Due to the imaging requirements of this population, an indepth clinical assessment via virtual visit is not possible unless diagnostics can be obtained. In certain circumstances, an US may be completed, and a phone call organized to discuss the results; this option should be explored for any children who would otherwise have a postponed in-person visit. A detailed history and review of the presence and duration of symptoms, as well as UTIs, passing of stone fragments, and pain episodes will help prioritize the urgency of clinic visits.

\section{Investigations to be obtained prior to assessment}

For new urgent consultation requests/referrals, a baseline US to assess the severity of $\mathrm{HN}$ or stone burden is essential. In addition, laboratory investigations, such as renal function markers, electrolytes, and initial stone metabolic workup, may also be indicated.

\section{Management}

When possible, medical management should be optimized, including adequate hydration and pain management. For stones that may pass spontaneously (i.e., $<5 \mathrm{~mm}$, distal ureteral location) alpha-blocker therapy may be considered to facilitate this. Families should be advised to collect any stones expelled. For those that meet criteria for urgent intervention, stent placement, nephrostomy tube insertion, or pyeloplasty should be considered on a case-by-case basis.

\section{Recurrent UTIs and VUR}

\section{(Authors: Dr. Peter Wang; Dr. Sumit Dave)}

\section{Background}

VUR has a prevalence in the general population of $0.4-$ $1.8 \%$, which increases to $30-50 \%$ in children with UTI. ${ }^{15,16}$ Treatment is aimed at reducing the potential morbidities of recurrent UTI and renal scarring. ${ }^{17,18}$ During COVID-19, when possible, the indications for obtaining imaging of VUR patients may follow the guidelines from the U.K.'s National Institute for Health and Care Excellence (NICE). NICE recommends an US within six weeks after an initial febrile UTI for children $\leq 6$ months of age, and US within six weeks for the second febrile UTI in children $>6$ months of age. The urgency of performing a VCUG after UTI is multifactorial, however, it can often be safely delayed by keeping the child on CAP.

\section{Conditions to screen for and red flag signs/symptoms}

Secondary causes of VUR (such as neurogenic bladder and PUV, which may be suspected if there is US evidence of $\mathrm{HN}, \mathrm{HUN}$, bladder abnormalities, or high post-void residuals [PVR]).

\section{Triage recommendations}

\section{Urgent, in-person clinic assessment}

- Suspicion of VUR being due to one of the red flags mentioned above

- If VUR is associated with severe HN or HUN and a secondary obstruction is suspected

- Postoperative patients with potential complications

\section{Postponed in-person clinic assessment}

- New referrals requiring imaging

- Recurrent UTIs

- Stable postoperative followup

- Routine surveillance with US

Most children with VUR can be safely postponed for in-person visits if CAP has been appropriately initiated. If breakthrough UTIs occur, a change of CAP via virtual visit may be considered.

\section{Virtual visit/telephone encounter}

This condition is amenable to normal triaging practices, as virtual visits may be used for both an initial consultation and followup. It is especially useful if imaging has been performed and available for review prior to the visit. During the initial consultation, it is important to determine whether VUR is primary vs. secondary. For primary VUR, age, sex, circumcision status, CAP use, number and severity of UTIs, whether they were febrile, and whether VUR was diagnosed during the workup of $\mathrm{HN}$ or after UTI should be determined. For secondary VUR, in addition to these patient variables, the presence of bladder and bowel dysfunction (BBD) is an important factor to elicit. 
Keefe et al

\section{Investigations to be obtained prior to assessment}

Documentation of UTIs, including urinalysis and cultures, which may guide CAP selection, as well as associated imaging (US and VCUG).

\section{Management}

CAP is the initial treatment option for most patients, and several risk stratification variables guide its usage. ${ }^{19}$ It may be a safe option while pandemic restrictions are in place and clinic assessments not possible. Breakthrough UTIs may be initially managed by changing the CAP.

\section{Urgent or life-threatening conditions: End-stage renal disease and genitourinary neoplasms}

(Authors: Dr. Armando Lorenzo; Dr. Daniel Keefe)

\section{Background}

The management of end-stage renal disease (ESRD) from a urological perspective involves the placement and maintenance of peritoneal dialysis (PD) catheters, pre-renal transplant assessment and counselling, and the transplant procedure itself. ${ }^{20}$ Many centers have placed renal transplantation on hold during the pandemic, making pre-transplant urological assessments less urgent than prior to COVID-19. However, PD catheter insertion or maintenance may qualify for urgent consultation to minimize the risk of requiring emergency placement of hemodialysis catheters. Home PD, for those capable, would undoubtedly reduce the frequency of hospital visits compared to clinic-based hemodialysis. ${ }^{21}$ Neoplasms of the genitourinary tract, while uncommon in children, will require urgent assessment despite the significant limitations of in-person visits during the pandemic. ${ }^{22}$

\section{Conditions to screen for and red flag signs/symptoms}

- Patients with severe hypertension on multiple medications or massive proteinuria may require pre-transplant nephrectomies; these can be selectively performed on an urgent basis

- Palpable masses or suspicious lesions on imaging should trigger a phone consultation with the on-call urologist for further instructions and suggestions

\section{Triage recommendations}

\section{Urgent, in-person clinic assessment}

- Palpable masses or suspicious lesions on imaging

- High-risk patients, who may experience significant morbidity if transplantation is delayed, should complete pretransplant workup in anticipation of a transplant once restrictions are lifted

- ESRD patients may be in the hospital for other visits or dialysis, making an in-person visit possible

\section{Postponed in-person clinic assessment}

- Stable ESRD patients who require imaging for pre-transplant assessment and/or physical examination

- Postoperative patients who are stable with no concerns (i.e., orchiectomy, partial orchiectomy, nephrectomy with at least one stable followup)

\section{Virtual visit/telephone encounter}

Virtual visits for pre-transplant assessments are feasible if all the diagnostics, such as laboratory investigations and diagnostic imaging, have been completed. The virtual visit can allow appropriate pre-transplant history, however, the physical exam should be completed in person preoperatively. Neoplasms require in-person assessments, however, discussion of results of diagnostics, such as imaging and pathology, may be suitable for virtual consults.

\section{Investigations to be obtained prior to assessment}

In ESRD and pre-transplant evaluations, standard laboratory investigations should be available for review from the nephrology/transplant team. In addition, a renal US with doppler to assess vascular patency is required prior to the visit. Patients with CAKUT may benefit from a VCUG. If referring providers suspect a malignancy, baseline laboratory investigations should be initiated (basic hematology, chemistry, and relevant tumor markers), as well as appropriate imaging (US). Obtaining these investigations should not delay the referral.

\section{Management}

ESRD management will be dictated by the urgency of requiring dialysis. PD is preferred over hemodialysis in most cases to facilitate home PD. Renal transplantation will be deferred until restrictions are lifted, however, pre-transplant evaluations should continue to expedite transplantation in the future. Children with neoplasms will require additional imaging, as well as assessment by the oncology team. 
Managing pediatric urology conditions during COVID-19

Management will be dictated by the specific malignancy, stage, and grade. Multidisciplinary oncology discussion via teleconferencing can help determine appropriate management based on available investigations and pathology.

\section{Penile pathology (hypospadias/phimosis/paraphimosis) (Authors: Dr. Mélise Keays; Dr. Luis Guerra)}

\section{Background}

Hypospadias is a common penile birth defect occurring in one in 200 live male births. The majority of cases will undergo elective surgical correction at 6-18 months of age. ${ }^{23}$ Physiological phimosis represents a normal finding at birth, ${ }^{22}$ with retractability increasing progressively with age. ${ }^{23}$ Pathological phimosis may occur due to lichen sclerosis, symptoms (recurrent balanoposthitis, skin-cracking, forceful retraction) or failure to retract after puberty. Paraphimosis occurs when the foreskin is retracted proximal to (or behind) the glans and cannot be reduced.

\section{Conditions to screen for and red flag signs/symptoms}

- Disorder of sexual differentiation (DSD)

- Proximal hypospadias with associated cryptorchidism in a newborn

- Obstructive urinary symptoms/UTI

- Paraphimosis

- Balanoposthitis

- Obstructive urinary symptoms

- New-onset urinary incontinence

- Presence of hair tourniquet on the penis

- Urinary retention

\section{Triage recommendations}

\section{Urgent, in-person clinic assessment}

- Newborns with possible DSD with associated cryptorchidism who require a physical exam, metabolic, endocrine, and genetic evaluation, as well as abdominal US

- Uncorrected hypospadias or postoperative cases with obstructive urinary symptoms (urinary retention or UTI) or foreskin/scrotal cellulitis

- Postoperative patients requiring an in-clinic procedure, such as a stent/dressing removal

- Pathological phimosis with retention or infection

- Paraphimosis medical emergency

\section{Postponed in-person clinic assessment}

- New hypospadias consultations and elective surgical repairs

- Routine postoperative followups

- Physiologic phimosis may not require urological consultation and early management should be deferred to the primary care provider

- Suspected pathological phimosis with no voiding concerns or mildly symptomatic cases

\section{Virtual visit/telephone encounter}

Many patients with penile concerns will require a physical exam to make any recommendations for surgical correction or identify postoperative complications. In all cases, a virtual assessment can help identify patients in need of more urgent care. A thorough history, including voiding patterns, history of prior prepuce retraction, episodes of balanitis or UTI, prior treatments with topical corticosteroids, medical history, and family history can be elicited. In some circumstances, video consultation or genital photography through a secure server can be used with patient and family consent. The use of this technology should be guided by the comfort level of patients and parents, and consent obtained from the child if they are old enough to do so. However, there may be video or photo quality issues that impair the ability to make a correct diagnosis and it is the authors' opinion that an in-person physical exam should be performed prior to making surgical recommendations.

\section{Investigations to be obtained prior to assessment}

Secure photographs of hypospadias/penile concerns after documentation of patient consent for virtual visits. No investigations required for isolated hypospadias.

\section{Management}

Most hypospadias consultations and primary repairs will be deferred until pandemic precautions have been lifted. Primary care providers or obstetricians diagnosing hypospadias in otherwise healthy boys with a normal testicular exam can reassure parents after spontaneous voiding is witnessed that the isolated condition does not require urgent assessment. Asymptomatic, physiological phimosis is considered normal and does not require medical treatment or specialist consultation. ${ }^{24}$ Patients with bothersome symptoms or evidence of early lichen sclerosus should begin medical therapy with topical corticosteroid. Simple balanitis can be managed at home with avoidance of irritants, regular baths, and topical antibiotic ointment. More advanced cases will require oral antibiotics. 
Inguinal pathology (hernia and hydrocele)

(Authors: Dr. Linda Lee; Dr. Allen Hayashi)

\section{Background}

Pediatric inguinal hernias and hydroceles form due to a patent processus vaginalis (PPV). The incidence of an inguinal hernia is $0.8-5 \%$ in full-term infants, with a higher incidence in premature infants. ${ }^{24}$ The overall risk of incarceration is $6-18 \%$ among children and the risk is highest, up to $30 \%$, in the first year of life. ${ }^{25}$ The goal of surgical management is to reduce the risk of incarceration and alleviate symptoms.

\section{Conditions to screen for and red flag signs/symptoms}

- Signs of incarceration (irreducible swelling, inconsolable child, nausea, vomiting, abdominal distension, erythema over the groin and scrotal skin)

- Scrotal swelling, including signs of vascular compromise to the testicle from incarcerated hernia compression or testicular torsion

\section{Triage recommendations}

\section{Urgent, in-person clinic assessment}

- Inguinal hernias with signs of incarceration

- Recently manually reduced incarcerated inguinal hernias due to $15 \%$ chance of developing recurrent incarceration if delayed $>5$ days $^{26}$

- Premature infants/newborns with inguinal hernias

- Postoperative patients with complications

\section{Postponed in-person clinic assessment}

- Older patients with inguinal hernias

- Isolated asymptomatic hydroceles

\section{Virtual visit/telephone encounter}

The clinical history is important, as the ability to examine the child is lost, and should include the duration of symptoms, history of painful episodes, presence of palpable bulge, and fluctuation in swelling. If possible, observing the cooperative child in the standing position with the parents identifying where they may have seen a hernia or hydrocele is often very useful. Parents can be educated regarding "red flags" and may be able to check for reducibility of the hernia and transillumination.

\section{Investigations to be obtained prior to assessment}

The diagnosis is based on clinical history and physical examination. If the clinical examination is equivocal or other diagnoses are being considered (e.g., inguinal or scrotal mass), US is a useful adjunct.

\section{Management}

Surgical intervention is the required management for inguinal pathologies. Emergent cases, such as incarcerated hernias, will proceed as they did prior to the pandemic. All other cases should be prioritized according to symptoms

\section{Bladder and bowel dysfunction \\ (Author: Dr. Joana dos Santos)}

\section{Background}

BBD represents approximately $40 \%$ of pediatric urology consults ${ }^{27,28}$ and is a functional elimination disorder that describes an umbrella of lower urinary tract symptoms associated with constipation and/or encopresis. Much of the primary management of BBD can be accomplished by community providers. Due to the non-surgical and non-urgent nature of $\mathrm{BBD}$, it is the pediatric urological condition most amenable to virtual visits and these visit types may be considered beyond the pandemic in an attempt to minimize clinic volumes and congestion.

\section{Conditions to screen for and red flag signs/symptoms}

- Central neurological causes of BBD (new-onset seizures, developmental delay, regression of milestones)

- Neuropathic bladder due to spinal cord abnormalities (including associated lower limb abnormalities, abnormal lower back exam)

- Endocrine/renal (headache, polyuria/polydipsia, hypertension)

- Urinary retention

Triage recommendations

Urgent, in-person clinic assessment

- If there is any suspicion of the red flags mentioned above

\section{Postponed in-person clinic assessment}

- $\quad \geq 2$ febrile UTI with positive urine cultures (catheter sample in non-toilet-trained or midstream in toilet-trained children) 
- Evidence of $\geq 6$ months of unsuccessful bladder retraining

- Abnormal US (HN, renal scarring, cortical thinning)

- Stable renal function

\section{Virtual visit/telephone encounter}

For the vast majority of children and adolescents with BBD, virtual consults represent a great opportunity to expedite a thorough assessment and initiate treatment, while practicing social distancing. For new BBD referrals with confirmed febrile UTIs and/or abnormal upper tracts, CAP should be considered until a specialized assessment has been secured. The initial assessment must include a full history: voiding symptoms; bowel history; febrile UTIs; toilet-training process; known urinary tract malformations; potential red flags. Social support, issues at home and developmental/behavioral issues with the child should be elicited. Refractory/complex BBD patients on medication and/or alternative therapies are candidates for virtual consults, as investigations have already been completed and adjustments to therapies can be made remotely.

\section{Investigations to be obtained prior to assessment}

A 48-hour voiding diary (voided volumes, accidents, bowel movements, fluid intake), dysfunctional voiding scoring system (DVSS or Vancouver) and Bristol Stool Scale should be completed at baseline and followups. Urinalysis, urine cultures, and relevant lab and imaging results should be reviewed.

\section{Management}

Urotherapy should be initiated, ${ }^{27,29-31}$ the bladder training video $^{32}$ reviewed, and children with recurrent febrile UTIs may be started on CAP. ${ }^{33}$ Additional interventions for those who have failed conservative management, such as medications, may be considered. ${ }^{34}$

\section{Ambiguous genitalia and differences in sexual differentiation}

\section{(Authors: Natasha Brownrigg, NP; Dr. Luis Braga)}

\section{Background}

Any type of genital ambiguity in a newborn can provoke major psychological distress for parents ${ }^{35}$ and calls for prompt multidisciplinary evaluation and care. ${ }^{36,37}$ The umbrella term, $\mathrm{DSD}$, is used to describe a heterogeneous group of congenital anomalies affecting the development of chromosomal, gonadal, or anatomical sex. ${ }^{36,37}$ DSD has an incidence of one in 4500-5000 live births. ${ }^{36}$ This umbrella term was proposed by the Chicago consensus statement in 2006, but since that time, there has been a lack of agreement in its use. Some patients and families still prefer terms like intersex, while others prefer DSD, but most prefer to be identified by their individual condition. Placing everyone under a single umbrella term is risky and because it is so broad, it does not assist in clinical care. This is particularly true for $46, \mathrm{XX}$ females with $\mathrm{CAH}$ and $46, X Y$ males with hypospadias, where discordant gender identity is rarely an issue. During the COVID-19 pandemic, as with any other medical specialty, certain aspects of DSD care may have to be postponed to adapt to this new reality. However, the assessment of DSD in newly referred patients should not be delayed.

\section{Conditions to screen for and red flag signs/symptoms}

- Evidence of salt-wasting congenital adrenal hyperplasia (CAH)

- Enlarged clitoris, posterior labial adhesions, inguinal/ labial mass in genitalia appearing female ${ }^{37}$

- Bilateral undescended testes, micropenis, isolated perineal hypospadias, or hypospadias with undescended testis in genitalia appearing male ${ }^{37}$

- Obvious genital ambiguity (i.e., cloacal exstrophy) $)^{37}$

- Discordance between prenatal karyotype and phenotype $^{37}$

\section{Triage recommendations}

\section{Urgent in-person clinic assessment}

- New suspicion of DSD in a newborn or infant. The primary goal is to identify life-threatening conditions requiring immediate medical care (i.e., salt-wasting $\mathrm{CAH}$ with a risk of hypovolemic shock); gender assignment should only be made after a thorough evaluation by the multidisciplinary team ${ }^{36,37}$

- Postoperative patients reporting complications

\section{Postponed in-person clinic assessment}

- Stable patients being routinely followed with a confirmed DSD diagnosis and plan of care

\section{Virtual visit/telephone encounter}

For children with a suspicion of DSD later in childhood (previously unrecognized ambiguous genitalia, inguinal hernia in a female, delayed or incomplete puberty, virilization in a female, primary amenorrhoea, breast development in males, or gross cyclic hematuria in males ${ }^{37}$ ), a virtual encounter permits the provider to obtain a detailed history and arrange additional investigations and/or referrals to other subspecial- 
ties. Prenatal consultations for suspected DSD can be carried out after a review of appropriate testing and imaging. Patients whose surgery is delayed may be appropriate for a virtual visit for surgical counselling and planning, keeping in mind that the timing of surgery is controversial. ${ }^{37,38}$ In addition, even though the risk of malignancy is higher in cases of streak gonads, this risk is insignificant before puberty, therefore, gonadectomy may be postponed until the pandemic is over.

\section{Investigations to be obtained prior to assessment}

Karyotype; abdominal and pelvic US; biopsies of gonadal tissue; genetic testing; laboratory investigations, including serum 17-hydroxyprogesterone; testosterone; gonadotropins (i.e., follicle-stimulating hormone [FSH], luteinizing hormone [LH]); anti-Mullerian hormone; serum electrolytes; urinalysis; human chorionic gonadotropin (hCG) and adrenocorticotropic hormone (ACTH) stimulation tests; urinary steroid analysis. ${ }^{37}$

\section{Management}

A multidisciplinary approach should be taken with all children receiving a gender assignment. Surgeons must outline surgical plans and discuss potential complications related to having early surgery or postponing the procedure. In cases of severe virilization (Prader III, IV, and V), surgery may be offered based on recommendations from the 2006 consensus statement. Functional outcome is the primary focus and psychosocial care should be offered. ${ }^{37}$

\section{Neurogenic lower urinary tract dysfunction \\ (Authors: Dr. Soojin Kim; Dr. Andrew MacNeily)}

\section{Background}

The International Children's Continence Society (ICCS) endorses the definition of lower urinary tract dysfunction (LUTD) as an abnormal or difficult function of the bladder and urethra. ${ }^{39,40}$ Specifically for neurogenic LUTD, the dysfunction may be due to spinal cord injury, spinal dysraphism, brain or spinal tumor, cerebral palsy, transverse myelitis, iatrogenic insult, and non-neurogenic neurogenic causes. ${ }^{41}$ During the COVID-19 pandemic, patients with neurogenic LUTD and their caregivers may have many questions and concerns, however, the Spinal Bifida Association reported that, in general, individuals with spinal dysraphism are not at increased risk of contracting COVID-19 or suffering severe illness from the virus. ${ }^{42}$

\section{Conditions to screen for and red flag signs/symptoms}

- Atypical BBD presentation (new onset of retention, encopresis, and/or lower limb abnormalities)

- Known patients with changes in voiding patterns, encopresis, and/or lower limb changes

- Changes to upper tracts on US (HN, HUN), bladder abnormalities

- Febrile UTIs

\section{Triage recommendations}

\section{Urgent, in-person clinic assessment}

- Newborns with spinal dysraphism

- Sudden onset of changes to voiding, either retention or incontinence

- Postoperative complications

- Symptomatic kidney/bladder stones

\section{Postponed in-person clinic assessment}

- Established bladder emptying regimen

- Properly treated UTIs

- Routine surveillance

- Stable postoperative patients

\section{Virtual visit/telephone encounter}

While this population typically has imaging associated with their visits, virtual followups can be used to ensure there are no changes to status since the last visit. Changes to clean intermittent catheterization $(\mathrm{CIC})$ schedules, starting or discontinuing medications, and recommending CAP can all be completed virtually. For new patients with neurogenic LUTD, an initial virtual consult can help to identify voiding patterns, bowel patterns, and symptoms, such as UTIs and episodes of retention.

\section{Investigations to be obtained prior to assessment}

Renal US and baseline renal function laboratory studies may be carried out prior to assessment. A voiding and bowel diary, including catheter volumes, may help estimate bladder capacity, determine ideal frequency of CICs, and optimize bowel regimen. Other potential investigations, such as nuclear scans, VCUGs, and urodynamics (UDS), should be indicated by urology clinicians and can usually be done on a non-urgent basis. ${ }^{43,44}$ 
Managing pediatric urology conditions during COVID-19

\section{Management}

Establishment of effective bladder drainage is the initial main priority to protect upper tract functional compromise. This is generally accomplished with $\mathrm{CIC}$, as well as initiation of appropriate medications, including anticholinergics. Patients with recurrent UTIs may benefit from CAP until more detailed assessment or bladder management is optimized.

\section{Concerns regarding future fertility (including undescended testicles, varicoceles)}

(Authors: Dr. Anne-Sophie Blais; Dr. Katherine Moore;

\section{Dr. Stéphane Bolduc)}

\section{Background}

Undescended testicles (UDT) and varicoceles are common testicular conditions encountered in the pediatric urology clinic. The risks of short-term delay in the management of these conditions are minimal but prolonging delayed investigation or treatment may lead to worrisome consequences, including infertility, hypogonadism, and concern for increased risk of malignancy. Severe germ cell loss has been identified in testicles that remain undescended by the age of two years. ${ }^{45}$ Histological effects of varicoceles are still not well-understood in adolescents, but there is adult literature showing altered testicular growth and decreased spermatogenesis affecting fertility potential. ${ }^{46,47}$ Delayed diagnosis and treatment of these conditions as a result of pandemic restrictions may lead to parental concern.

\section{Conditions to screen for and red flag signs/symptoms}

- UDT: Bilateral non-palpable especially if associated with hypospadias; these may represent CAH/DSD

- Varicocele: Acute onset, isolated right-sided, non-reducible in the recumbent position; these may be secondary to compressive retroperitoneal/abdominal masses

- Suspected torsion (usually via the ED)

\section{Triage recommendations}

\section{Urgent, in-person clinic assessment}

- Newborns with bilateral UDT

- UDT associated with penile anomalies

- Right-sided varicoceles

- Varicoceles in prepubescent children

- Neonatal torsion

\section{Postponed in-person clinic assessment}

- New referrals for UDTs and left-sided varicoceles

- Postoperative patients without concerns

\section{Virtual visit/telephone encounter}

An essential component of the assessment of children with UDT and varicoceles is the physical examination. ${ }^{48}$ Consultation should be postponed for an in-person clinic assessment. However, virtual/telephone encounters can help the clinician to distinguish retractile from UDT (which may change rebooking priority) and to identify patients who may need further investigations before assessment. A patient (or parent) self-exam may help to document testicular position, testicular volume discrepancy, grade of varicocele, and integrity of the contralateral testicle. For centers offering fertility preservation for oncology patients, counselling and surgical discussion can be carried out with virtual visits.

\section{Investigations to be obtained prior to assessment}

For bilateral non-palpable testes (NPT) and patients with UDT and proximal hypospadias, a genetic and endocrine evaluation should be considered. ${ }^{48}$ Moreover, newborns with bilateral NPT should undergo an endocrine evaluation to rule out $\mathrm{CAH}$. An abdominal US should be obtained for prepubescent varicoceles, right-sided varicoceles, and/or varicoceles that do not fluctuate with Valsalva. US assessment for UDT in the absence of any red flags is not indicated.

\section{Management}

Management of these issues is surgical, and the scheduling of procedures will depend on surgical volumes and backlog once restrictions are lifted.

\section{Bladder and cloacal exstrophy}

(Authors: Dr. Landan MacDonald; Dr. Dawn MacLellan; Dr. Peter Anderson; Dr. Rodrigo Romao)

\section{Background}

A trend to delay the repair of classic bladder exstrophy by a few months after birth, as opposed to the traditional approach of repairing it in the neonatal period, has been described in the pre-pandemic era in high-volume centers ${ }^{49}$ and is aligned with the recently published European Association of Urology (EAU) COVID-19 guideline. ${ }^{22}$ For cloacal exstrophy patients, management will be determined on a case-by-case basis. The presence of associated anomalies, such as myelomenin- 
Keefe et al

gocele, and gastrointestinal tract status will dictate the need for an urgent intervention in the neonatal period.

\section{Conditions to screen for and red flag signs/symptoms}

- Symptomatic bladder stones

- Febrile UTIs

- Renal function compromise due to obstruction (postrepair)

\section{Triage recommendations}

\section{Urgent, in-person clinic assessment}

- Newborns with bladder/cloacal exstrophy

- Postoperative complications

- Suspicion of bladder stones

\section{Postponed in-person clinic assessment}

- Routine followups

- UTIS

- Management of continence issues

- Fertility discussion

\section{Virtual visit/telephone encounter}

Routine surveillance can be completed via virtual visits; management of continence issues and discussion surrounding fertility concerns are also ideal for virtual consults. Surgical counselling may also be completed via virtual visit, as well as changes to current continence medications and regimens.

\section{Investigations to be obtained prior to assessment}

US of kidneys prior to initial assessment; documentation of any UTIs

\section{Management}

If the neonate is stable, there is no need to transfer to a specialized center until mother can also be discharged. Communication between the neonatal care unit and pediatric urology team is needed to counsel parents and provide instructions on care of the bladder plate, including coverage with a Tegaderm ${ }^{\circledR}$ or plastic wrap, silk tie on umbilical cord, and avoidance of prophylactic antibiotics prior to bladder closure to avoid antimicrobial resistance.

\section{Recommendations for minimizing clinic congestion after the COVID-19 pandemic through optimization of telemedicine}

Canada was an early pioneer in the realm of remote provision of care, with Dr. Maxwell House delivering telemedicine care to remote regions in Newfoundland dating back to the 1970s. Our vast geography is a great impetus to continue to expand this domain of healthcare delivery.

The COVID-19 pandemic has provided unprecedented opportunity for clinicians to implement strategies to reduce clinic volumes. This endeavor has led to a substantial increase in telemedicine and virtual visits. Expert opinion from across the country favors expanding the use of telemedicine in clinics, as highlighted in this document, even once the current pandemic resolves. The panel would suggest that patients ideal for telemedicine would be those where the patient and family have the means and motivation to be assessed in this fashion, an available local physician or healthcare provider that can be engaged as a partner, and accessibility to obtain imaging outside of the hospital environment that can be reviewed and interpreted by the pediatric urology clinician during the visit if required. Although beyond the scope of this commentary, we would direct healthcare providers participating in telemedicine to the guidelines outlined by the Canadian Medical Association and the American Telemedicine Association. ${ }^{50,51}$

Conditions that can be managed almost exclusively with virtual visits as long as the above conditions are met include:

- BBD

- Prenatal CAKUT parental counselling

- Stable, mild, and moderate HN

- VUR with no UTIs and stable imaging

- Neurogenic LUTD with stable imaging, established bladder and bowel routines, and no infections

- Selective postoperative patients without complication concerns (circumcisions, inguinal hernias, orchidopexy)

\section{Conclusions}

The COVID-19 pandemic has caused a drastic change in our ability to provide care for pediatric urology patients. Urgent/ emergent life- and limb-threatening conditions continue to require immediate urological assessment. However, many pediatric urology conditions are amenable to delayed in-person assessment or virtual encounters. As we move through this pandemic and once restrictions are lifted, we will be responsible for appropriately triaging the backlog of patients. In the future, there may be similar unforeseen circumstances and these guidelines can help as a basis for management. This document, created by experts in pediatric urology in 
Managing pediatric urology conditions during COVID-19

Canada, is aimed to guide stratification of patients according to urgency and suggest appropriate investigations to initiate while awaiting specialist assessment.

Competing interests: Dr. Bolduc has been a principal investigator for clinical trials supported by Astellas and Pfizer. Dr. Moore has been an advisory board member for Pfizer; has received speaker honoraria for Duchesnay and Hollister; and has been an investigator for clinical trials supported by Astellas and Pfizer. The remaining authors reports no competing personal or financial interests related to this work.

This paper has been peer-reviewed.

\section{References}

1. WHO Director-General's opening remarks at the media briefing on COVID-19. April 22, 2020. Available at: https://www.who.int/dg/speeches/detail/who-director-general-s-opening-remarks-at-the-media-briefingon-covid-19-22-apri-2020. Accessed April 26, 2020.

2. Fisher D, Heymann D: Q\&A: The novel coronavirus outbreak causing COVID-19. BMC Med 2020;18:57. https://doi.org/10.1186/s12916-020-01533-w

3. Ferreira $R$, McGrath $M$, Wang $Y$, et al. How to prioritize urological surgeries during epidemics: Lessons learned from the Toronto SARS outbreak in 2003. Can Urol Assoc J 2020; 14:E159-60. https://doi.org/10.5489/cuai.6551

4. Beck CA, Beran DB, Biglan KM, et al. National randomized controlled trial of virtual house calls for Parkinson disease. Neurology 201789: 1152-61. https://doi.org/10.1212/WNL.0000000000004357

5. Ware P, Ross HJ, Cafazzo JA, et al. Outcomes of a heart failure telemonitoring program implemented as the standard of care in an outpatient heart function clinic: Pre-test-post-test pragmatic study. J Med Internet Res 2020;22:e16538. https://doi.org/10.2196/16538

6. Dixon RF, Stahl JE. Virtual visits in a general medicine practice: A pilot study. Telemed J E Health 2008;14:525-30. https://doi.org/10.1089/tmi.2007.0101

7. Sultan AA, Acuña AJ, Samuel LT, et al. Utilization of telemedicine virtual visits in pediatric spinal deformity patients: A comparison of feasibility and patient satisfaction at a large academic senter. J Pediatr Orthop 2020. [Epub ahead of print]. https://doi.org/10.1097/BP0.0000000000001553

8. Connor MJ, Miah S, Edison MA, et al. Clinical, fiscal and environmental benefits of a specialist-led virtual ureteric colic clinic: A prospective study. BJU Int 2019;124:1034-9. https://doi.org/10.1111/bju.14847

9. Miah $S$, Dunford $C$, Edison $M$, et al. A prospective clinical, cost, and environmental analysis of a clinician-led virtual urology clinic. Ann R Coll Surg Engl 2019;101:30-4. https://doi.org/10.1308/ rcsann.2018.0151

10. Chiodini B, Ghassemi $M$, Khelif $K$, et al. Clinical outcome of children with antenatally diagnosed hydronephrosis. Front Pediatr 2019;7:103. https://doi.org/10.3389/fped.2019.00103

11. Arora M, Prasad A, Kulshreshtha R, et al. Significance of third trimester ultrasound in detecting congenital abnormalities of kidney and urinary tract - a prospective study. J Pediatr Urol 2019;15:334-40. https://doi.org/10.1016/i.jpurol.2019.03.027

12. Lee RS, Cendron M, Kinnamon DD, et al. Antenatal hydronephrosis as a predictor of postnatal outcome: A meta-analysis. Pediatrics 2006;118:586-93. https://doi.org/10.1542/peds.2006-0120

13. Capolicchio J-P, Braga LH, Szymanski KM: Canadian Urological Association/Pediatric Urologists of Canada guideline on the investigation and management of antenatally detected hydronephrosis. Can Urol Assoc J 2018;12: 85-92. https://doi.org/10.5489/cuai.5094

14. Silay MS, Undre S, Nambiar AK, et al. Role of antibiotic prophylaxis in antenatal hydronephrosis: A systematic review from the European Association of Urology/European Society for Pediatric Urology Guidelines Panel. J Pediatr Urol 2017;13:306-15. https://doi.org/10.1016/i.jpurol.2017.02.023

15. Baker R, Maxted W, Maylath J, et al. Relation of age, sex, and infection to reflux: Data indicating high spontaneous cure rate in pediatric patients. J Urol 1966;95:27-32. https://doi.org/10.1016/ S0022-5347(17)63403-7

16. Sargent MA. What is the normal prevalence of vesicoureteral reflux? Pediatr Radiol 2000;30:587-93 https://doi.org/10.1007/s002470000263

17. Smellie JM. Reflections on 30 years of treating children with urinary tract infections. J Urol 1991;146:6658. https://doi.org/10.1016/S0022-5347(17)37889-8

18. Ransley PG, Risdon RA: The pathogenesis of reflux nephropathy. Contrib Nephrol 1979;16:90-7. https://doi.org/10.1159/000402880
19. Wang ZT, Wehbi E, Alam Y, et al. A re-analysis of the RIVUR trial using a risk classification system. J Urol 2018;199:1608-14. https://doi.org/10.1016/i.juro.2017.11.080

20. Palmer B, Kropp B: Urologic evaluation and management of pediatric kidney transplant patients. Urol Clin North Am 2018;45:561-9. https://doi.org/10.1016/i.ucl.2018.06.004

21. Wilkie M, Davies S. Peritoneal dialysis in the time of COVID-19. Perit Dial Int 2020. [Epub ahead pf print]. https://doi.org/10.1177/0896860820921657

22. Quaedackers JSLI, Stein R, Bhatt N, et al. Clinical and surgical consequences of the COVID-19 pandemic for patients with pediatric urological problems. J Pediatr Urol 2020. [Epub ahead of print]. https://doi.org/10.1016/i.jpurol.2020.04.007

23. Manzoni G, Bracka A, Palminteri E, et al. Hypospadias surgery: When, what, and by whom? BJU Int 2004;94:1188-95. https://doi.org/10.1046/i.1464-410x.2004.05128.x

24. Bowling K, Hart N, Cox P, et al. Management of pediatric hernia. BMJ 2017;359. https://doi.org/10.1136/bmi.j4484

25. Esposito C, Escolino M, Turrà F, et al. Current concepts in the management of inguinal hernia and hydrocele in pediatric patients in laparoscopic era. Semin Pediatr Surg 2016;25:232-40. https://doi.org/10.1053/i.sempedsurg.2016.05.006

26. Koivusalo A, Pakarinen MP, Rintala RJ. Laparoscopic herniorrhaphy after manual reduction of incarcerated inguinal hernia. Surg. Endosc 2007;21:2147-9. https://doi.org/10.1007/s00464-007-9318-5

27. Santos ID, Lopes RI, Koyle MA. Bladder and bowel dysfunction in children: An update on the diagnosis and treatment of a common, but underdiagnosed pediatric problem. Can Urol Assoc J 2017;11:S64-72. https://doi.org/10.5489/cuaj.4411

28. Halachmi S, Farhat WA. Interactions of constipation, dysfunctional elimination syndrome, and vesicoureteral reflux. Adv Urol 2008:828275. hitps://doi.org/10.1155/2008/828275

29. Wang R, Kanani R, Mistry N, et al. Practical tips for pediatricians: Assessment and management of bladder and bowel dysfunction in the office. Paediatr Child Health 2020;25:136-8. hittps://doi.org/10.1093/ pch/pxz084

30. dos Santos J, Varghese A, Williams K, et al. Recommendations for the management of bladder bowel dysfunction in children. Pediatr Ther 2014;4. https://doi.org/10.4172/2161-0665.1000191

31. Allen $\mathrm{HA}$, Austin JC, Boyt MA, et al. Initial trial of timed voiding is warranted for all children with daytime incontinence. Urology 2007;69:962-5. https://doi.org/10.1016/i.urology.2007.01.049

32. Braga LH, Mandy R, Forough $F$, et al. Bladder training video vs. standard urotherapy for bladder and bowel dysfunction: A non-inferiority randomized, controlled trial. J Urol 2017;197:877-84. https://doi.org/10.1016/i.juro.2016.08.089

33. Peters $C A, S k o o g ~ S J$, Arant $B S$, et al. Summary of the AUA guideline on management of primary vesicoureteral reflux in children. J Urol 2010;184:1134-44. https://doi.org/10.1016/i.juro.2010.05.065

34. Cain MP, Wu SD, Austin PF, et al. Alpha blocker therapy for children with dysfunctional voiding and urinary retention. J Urol 2003;170:1514-5; discussion 1516-1517. https://doi.org/10.1097/01. ju.0000085961.27403.40

35. Suorsa KI, Mullins AJ, Tackett AP, et al. Characterizing early psychosocial functioning of parents of children with moderate to severe genital ambiguity due to disorders of sex development. J Urol 2015;194:173742. https://doi.org/10.1016/i.juro.2015.06.104

36. Kutney K, Konczal L, Kaminski B, et al. Challenges in the diagnosis and management of disorders of sex development. Birth Defects Res C Embryo Today 2016;108:293-308.

37. Hughes IA, Houk C, Ahmed SF, et al. Consensus statement on management of intersex disorders. J Pediatr Urol 2006;2:148-62. hitps://doi.org/10.1016/i.jpurol.2006.03.004

38. Braga LH, Pippi Salle JL. Congenital adrenal hyperplasia: a critical appraisal of the evolution of feminizing genitoplasty and the controversies surrounding gender reassignment. Eur J Pediatr Surg 2009;19:203-10. https://doi.org/10.1055/s-0029-1233490

39. Austin PF, Bauer SB, Bower W, et al. The standardization of terminology of lower urinary tract function in children and adolescents: Update report from the standardization committee of the International Children's Continence Society. Neurourol. Urodyn 2016;35:471-81. https://doi.org/10.1002/nau.22751

40. An International Continence Society (ICS) report on the terminology for adult neurogenic lower urinary tract dysfunction (ANLUTD). Available at: https://www-ncbi-nlm-nih-gov.myaccess.library.utoronto.ca/ pubmed/29149505. Accessed May 3, 2020.

41. Kavanagh A, Baverstock R, Campeau L, et al.: Canadian Urological Association guideline: Diagnosis, management, and surveillance of neurogenic lower urinary tract dysfunction - Executive summary. Can Urol Assoc J 2019;13. htrps://doi.org/10.5489/cuaj.6041

42. Spina Bifida Association. Coronavirus (COVID-19): What people with spina bifida need to know. Available at: https://www.spinabifidaassociation.org/news/coronavirus2020/. Accessed May 3, 2020.

43. Tanaka ST, Paramsothy P, Thibadeau J, et al. Baseline urinary tract imaging in infants enrolled in the UMPIRE protocol for children with spina bifida. J Urol 2019;201:1193-8. htrps://doi.org/10.1097/ JU.0000000000000141

44. Spina Bifida Association. Guidelines for the care of people with spina bifida. Available at: https://www.spinabifidaassociation.org/guidelines/. Accessed May 3, 2020. 
Keefe et al

45. Tasian GE, Hittelman AB, Kim GE, et al. Age at orchiopexy and testis palpability predict germ and Leydig cell loss: Clinical predictors of adverse histological features of cryptorchidism. J Urol 2009;182:704-9. https://doi.org/10.1016/i.juro.2009.04.032

46. 46. Hienz HA, Voggenthaler J, Weissbach L. Histological findings in testes with varicocele during childhood and their therapeutic consequences. Eur J Pediatr 1980;133:139-46. https://doi.org/10.1007/ BF00441582

47. Sharlip ID, Jarow J, Belker AM, et al. AUA best practice: Report on varicocele and infertility. Available at: https://www.auanet.org/Documents/education/clinical-guidance/Varicocele-Archive.pdf. Accessed May 21, 2020.

48. Braga LH, Lorenzo AJ, Romao RLP: Canadian Urological Association-Pediatric Urologists of Canada (CUA-PUC) guideline for the diagnosis, management, and followup of cryptorchidism. Can Urol Assoc J 2017;11:E251-60. https://doi.org/10.5489/cuai.4585
49. Borer JG, Vasquez E, Canning DA, et al. Short-ferm outcomes of the multi-institutional bladder exstrophy consortium: Successes and complications in the first two years of collaboration. J Pediatr Urol 2017;13:275.el-6. htrps://doi.org/10.1016/i.jpurol.2017.01.006

50. Krupinski EA, Bernhard J. Standards and guidelines in telemedicine and telehealth. Healthcare 2014;2:7493. https://doi.org/10.3390/healthcare2010074

51. Canadian Medical Association. Virtual care in Canada: Discussion Paper. 2019. Available at: https://www.cma.ca/sites/default/files/pdf/News/Virtual_Care_discussionpaper_v2EN.pdf. Accessed May 12, 2020.

Correspondence: Dr. Daniel T. Keefe, Hospital for Sick Children, Toronto, ON, Canada; daniel.keefe@sickkids.ca 